\title{
On the supremum of random Dirichlet polynomials
}

\author{
by \\ Mikhail Lifshits (St. Petersburg) and Michel Weber (Strasbourg)
}

\begin{abstract}
We study the supremum of some random Dirichlet polynomials $D_{N}(t)=$ $\sum_{n=2}^{N} \varepsilon_{n} d_{n} n^{-\sigma-i t}$, where $\left(\varepsilon_{n}\right)$ is a sequence of independent Rademacher random variables, the weights $\left(d_{n}\right)$ are multiplicative and $0 \leq \sigma<1 / 2$. Particular attention is given to the polynomials $\sum_{n \in \mathcal{E}_{\tau}} \varepsilon_{n} n^{-\sigma-i t}, \mathcal{E}_{\tau}=\left\{2 \leq n \leq N: P^{+}(n) \leq p_{\tau}\right\}, P^{+}(n)$ being the largest prime divisor of $n$. We obtain sharp upper and lower bounds for the supremum expectation that extend the optimal estimate of Halász-Queffélec,

$$
\mathbb{E} \sup _{t \in \mathbb{R}}\left|\sum_{n=2}^{N} \varepsilon_{n} n^{-\sigma-i t}\right| \approx \frac{N^{1-\sigma}}{\log N} .
$$

The proofs are entirely based on methods of stochastic processes, in particular the metric entropy method.
\end{abstract}

1. Introduction and main results. Let $\left\{d_{n}, n \geq 1\right\}$ be a sequence of real numbers. Let $s=\sigma+i t$ denote a complex number. The study of the supremum of the Dirichlet polynomials

$$
P(s)=\sum_{n=2}^{N} d_{n} n^{-s}
$$

over lines $\{s=\sigma+i t: t \in \mathbb{R}\}$ is naturally related to that of the corresponding Dirichlet series, via the abscissa of uniform convergence

$$
\sigma_{\mathrm{u}}=\inf \left\{\sigma: \sum_{n=2}^{\infty} d_{n} n^{-\sigma-i t} \text { converges uniformly over } t \in \mathbb{R}\right\},
$$

through the relation

$$
\sigma_{\mathrm{u}}=\limsup _{N \rightarrow \infty} \frac{\log \sup _{t \in \mathbb{R}}\left|\sum_{n=2}^{N} d_{n} n^{-i t}\right|}{\log N} .
$$

2000 Mathematics Subject Classification: Primary 30B50, 26D05; Secondary 60G17.

Key words and phrases: Dirichlet polynomials, Rademacher random variables, metric entropy method. 
One can refer to Bohr [B], Bohnenblust and Hille [BH], Helson [H], Hardy and Riesz [HR], Queffélec [Q3] for background and related results. This, of course, basically justifies investigation of the supremum of Dirichlet polynomials (see for instance Konyagin and Queffélec [KQ]).

The following classical reduction step enables one to replace a Dirichlet polynomial by some relevant trigonometric polynomial. To recall this reduction, we introduce the necessary notation. Let $2=p_{1}<p_{2}<\cdots$ be the sequence of all primes. If $n=\prod_{j=1}^{\tau} p_{j}^{a_{j}(n)}$, we write $\underline{a}(n)=\left\{a_{j}(n), 1 \leq j \leq \tau\right\}$. Let $\pi(N)$ denote, as usual, the number of prime numbers that are less then or equal to $N$. Finally, let $\mathbb{T}=[0,1[=\mathbb{R} / \mathbb{Z}$ be the torus. Fix $N$, put $\mu=\pi(N)$, and define, for $\underline{z}=\left(z_{1}, \ldots, z_{\mu}\right) \in \mathbb{T}^{\mu}$,

$$
Q(\underline{z})=\sum_{n=2}^{N} d_{n} n^{-\sigma} e^{2 i \pi\langle\underline{a}(n), \underline{z}\rangle}
$$

H. Bohr's famous observation ([Q1-3]) is that

$$
\sup _{t \in \mathbb{R}}|P(\sigma+i t)|=\sup _{\underline{z} \in \mathbb{T}^{\mu}}|Q(\underline{z})| .
$$

This indeed follows straightforwardly from Kronecker's theorem (see [HW, Theorem 442, p. 382]).

A parallel study is also developed for random Dirichlet polynomials and random Dirichlet series in the papers of Halász [Ha1-2], Queffélec [Q1-3], Bayart, Konyagin and Queffélec [BKQ], Kahane [K], Yu [Y1-3], Sun, Tian and $\mathrm{Yu}$ [STY], and Hedenmalm and Saksman [HS]. Such investigations concerning random Dirichlet series (as well as random power series) go back to earlier works of Hartman [Har], Clarke [C], Dvoretzky and Erdős [DE1-2], and Dvoretzky and Chojnacki [DC].

Let $\varepsilon=\left\{\varepsilon_{i}, i \geq 1\right\}$ (here and throughout) be a sequence of independent Rademacher random variables $\left(\mathbb{P}\left\{\varepsilon_{i}= \pm 1\right\}=1 / 2\right)$ defined on a basic probability space $(\Omega, \mathcal{A}, \mathbb{P})$.

Consider the random Dirichlet polynomials

$$
D(s)=\sum_{n=2}^{N} \varepsilon_{n} d_{n} n^{-\sigma-i t} .
$$

When $d_{n} \equiv 1$, some results about the suprema are known. If $\sigma=0$, then for some absolute constant $C$, and all integers $N \geq 2$,

$$
C^{-1} \frac{N}{\log N} \leq \mathbb{E} \sup _{t \in \mathbb{R}}\left|\sum_{n=2}^{N} \varepsilon_{n} n^{-i t}\right| \leq C \frac{N}{\log N} .
$$

This has been proved by Halász (see [Q2-3]). In [Q2-3] (see also [Q1] for a first result), Queffélec extended Halász's result to the range of values $0 \leq \sigma$ $<1 / 2$; he also provided a probabilistic proof of the original one, using Bern- 
stein's inequality for polynomials, properties of complex Gaussian processes and the sieve method introduced by Halász. He deduced that for some constant $C_{\sigma}$ depending on $\sigma$ only, and all integers $N \geq 2$,

$$
C_{\sigma}^{-1} \frac{N^{1-\sigma}}{\log N} \leq \mathbb{E} \sup _{t \in \mathbb{R}}\left|\sum_{n=2}^{N} \varepsilon_{n} n^{-\sigma-i t}\right| \leq C_{\sigma} \frac{N^{1-\sigma}}{\log N} .
$$

This in fact admits a stronger form

$$
C_{\sigma}^{-1} \leq \mathbb{E} \sup _{N \geq 2} \sup _{t \in \mathbb{R}} \frac{\left|\sum_{n=2}^{N} \varepsilon_{n} n^{-\sigma-i t}\right|}{N^{1-\sigma}(\log N)^{-1}} \leq C_{\sigma} .
$$

A proof is given at the end of Section 4 . We shall hereafter use and simplify Queffélec's probabilistic argument, notably reducing the proof of the upper bound part to the study of suitable real Gaussian processes (which can be easily reduced to a single one). Further, we will not use Bernstein's inequality, in contrast to both previous proofs. A simple metric entropy argument is indeed sufficient, making the proof entirely based upon stochastic processes methods.

By developing this approach, we will also study the case when the $d_{n}$ 's are not constant and random Dirichlet polynomials are supported by other sets than intervals of integers $[2, N]$. In this regard, we consider the following natural extension. For any integer $n>1$, let $P^{+}(n)$ denote the largest prime divisor of $n$. Let $1 \leq M<N$ be two positive integers and define

$$
S(N, M)=\left\{2 \leq n \leq N: P^{+}(n) \leq M\right\} .
$$

Since $S(N, N)=[2, N]$, these sets naturally generalize the notion of interval of integers. By using the standard notation

$$
\Psi(N, M):=\sharp S(N, M),
$$

$u=(\log N) / \log M$, we have $([\mathrm{T}$, Theorem 6, p. 405])

$$
\Psi^{*}(N, M):=\frac{\Psi(N, M)}{N}=\varrho(u)+\mathcal{O}\left(\frac{1}{\log y}\right),
$$

uniformly for $x \geq y \geq 2$, where $\varrho(u)$ is the Dickman function, the unique continuous function on $[0, \infty[$, having a derivative on $] 0, \infty[$ and such that

$$
\begin{array}{rlrl}
\varrho(v)=1 & & (0 \leq v \leq 1), \\
v \varrho^{\prime}(v)+\varrho(v-1) & =0 & & (v>1) .
\end{array}
$$

It is known that $\varrho(u)$ is a decreasing positive function and that $\log \varrho(u) \sim$ $-u \log u$ as $u \rightarrow \infty$. In other words, $\varrho$ decreases as fast as the inverse of the Gamma function. By setting $M=N^{\varepsilon}$ in (1.5) we see that $\Psi\left(N, N^{\varepsilon}\right) \sim$ $N \varrho\left(\varepsilon^{-1}\right)$ for any fixed $0<\varepsilon \leq 1$. 
In view of (1.5), we sometimes refer to $\Psi^{*}$ as a Dickman-type function.

Fix some positive integer $\tau \leq \pi(N)$, and recall that $p_{1}<p_{2}<\cdots$ is the sequence of primes. Put

$$
\mathcal{E}_{\tau}=\mathcal{E}_{\tau}(N)=\left\{2 \leq n \leq N: P^{+}(n) \leq p_{\tau}\right\} .
$$

Note that for $\mu=\pi(N)$ we have $\mathcal{E}_{\mu}=\{2, \ldots, N\}$.

The $\mathcal{E}_{\tau}$-based Dirichlet polynomials were already considered in [Q3]. One motivation for considering them, relating to the Rudin-Shapiro problem, will be explained later.

We begin with a result that contains both the above mentioned estimates (1.3) and (1.4).

THEOREM 1.1.

(a) (Upper bound) Let $0 \leq \sigma<1 / 2$. Then there exists a constant $C_{\sigma}$ such that for any integer $N \geq 2$,

$$
\mathbb{E} \sup _{t \in \mathbb{R}}\left|\sum_{n \in \mathcal{E}_{\tau}} \varepsilon_{n} n^{-\sigma-i t}\right| \leq \begin{cases}C_{\sigma} \frac{N^{1 / 2-\sigma} \tau^{1 / 2}}{(\log N)^{1 / 2}} & \text { if } N^{1 / 2} \leq \tau \leq N, \\ C_{\sigma} \frac{N^{3 / 4-\sigma}}{(\log N)^{1 / 2}} & \text { if } N^{1 / 2} / \log N \leq \tau \leq N^{1 / 2}, \\ C_{\sigma} N^{1 / 2-\sigma} \tau^{1 / 2} & \text { if } 1 \leq \tau \leq N^{1 / 2} / \log N .\end{cases}
$$

(b) (Lower bound) Let $0 \leq \sigma<1 / 2$. Then there exists a constant $C_{\sigma}$ such that for every $N \geq 2$,

$$
\mathbb{E} \sup _{t \in \mathbb{R}}\left|\sum_{n \in \mathcal{E}_{\tau}} \varepsilon_{n} n^{-\sigma-i t}\right| \geq \frac{C_{\sigma} N^{1 / 2-\sigma} \tau^{1 / 2}}{(\log \tau)^{1 / 2}} \cdot \Psi^{*}\left(\frac{N}{p_{\tau}}, p_{\tau / 2}\right)^{1 / 2} .
$$

Sharpness of the result. It is instructive to compare the lower and upper bounds obtained in Theorem 1.1.

Consider three cases, as in the upper bound of the theorem:

CASE I: $N^{1 / 2} \leq \tau \leq N$. Here the Dickman function vanishes from the lower bound and we have $\log \tau \sim \log N$. It follows from the theorem that

$$
C_{1}(\sigma) \frac{N^{1 / 2-\sigma} \tau^{1 / 2}}{(\log N)^{1 / 2}} \leq \mathbb{E} \sup _{t \in \mathbb{R}}\left|\sum_{n \in \mathcal{E}_{\tau}} \varepsilon_{n} n^{-\sigma-i t}\right| \leq C_{2}(\sigma) \frac{N^{1 / 2-\sigma} \tau^{1 / 2}}{(\log N)^{1 / 2}} .
$$

Thus our bounds are optimal.

CASE II: $N^{1 / 2} / \log N \leq \tau \leq N^{1 / 2}$. Again the Dickman function vanishes from the lower bound and we have $\log \tau \sim \log N$. Thus

$$
C_{1}(\sigma) \frac{N^{1 / 2-\sigma} \tau^{1 / 2}}{(\log N)^{1 / 2}} \leq \mathbb{E} \sup _{t \in \mathbb{R}}\left|\sum_{n \in \mathcal{E}_{\tau}} \varepsilon_{n} n^{-\sigma-i t}\right| \leq C_{2}(\sigma) \frac{N^{3 / 4-\sigma}}{(\log N)^{1 / 2}} .
$$


The ratio of the right and left hand sides satisfies

$$
1 \leq \frac{N^{1 / 4}}{\tau^{1 / 2}} \leq(\log N)^{1 / 2} .
$$

Thus a logarithmic gap appears.

CASE III: $1 \leq \tau \leq N^{1 / 2} / \log N$. Assume first that $\tau \geq N^{\varepsilon}$ for some fixed $\varepsilon>0$, necessarily with $\varepsilon<1 / 2$. Then the Dickman function produces in the lower bound just an extra constant depending on $\varepsilon$. We have

$$
C_{1}(\sigma, \varepsilon) \frac{N^{1 / 2-\sigma} \tau^{1 / 2}}{(\log \tau)^{1 / 2}} \leq \mathbb{E} \sup _{t \in \mathbb{R}}\left|\sum_{n \in \mathcal{E}_{\tau}} \varepsilon_{n} n^{-\sigma-i t}\right| \leq C_{2}(\sigma) N^{1 / 2-\sigma} \tau^{1 / 2} .
$$

The gap is still of the logarithmic order:

$$
1 \leq(\log \tau)^{1 / 2} \leq(\log N)^{1 / 2} .
$$

One should notice that an upper estimate $C N^{1 / 2-\sigma}(\tau \log \log N)^{1 / 2}$ slightly weaker than our bound in Case III was obtained in [Q3].

It is also worth mentioning that our approach to the lower bounds is very different from that in the preceding works [Q3], [KQ] based on deterministic estimates valid for any polynomial (see e.g. lower bound in (1.6) below). It would be interesting to check whether the optimisation of parameters in deterministic estimates enables this approach to compete with our lower bound on the whole range of $\tau$.

Unfortunately, if $\tau$ is relatively small, namely $\log \tau \ll \log N$, the gap between the upper and lower bounds in Theorem 1.1 becomes rather significant due to the small factor $\Psi^{*}$ in the lower bound. Our next result, although not optimal, shows that the presence of $\Psi^{*}$ is crucial.

TheOREM 1.2. Let $0 \leq \sigma<1 / 2$. Then there exists a constant $C_{\sigma}$ such that for any integer $N \geq 2$ and $\tau>\exp \left\{(\log \log N)^{2}\right\}$,

$$
\begin{aligned}
& \frac{N^{1 / 2-\sigma} \tau^{1 / 2} \Psi^{*}\left(N / p_{\tau}, p_{\tau / 2}\right)^{1 / 2}}{C_{\sigma}(\log \tau)^{1 / 2}} \\
& \quad \leq \mathbb{E} \sup _{t \in \mathbb{R}}\left|\sum_{n \in \mathcal{E}_{\tau}} \varepsilon_{n} n^{-\sigma-i t}\right| \leq C_{\sigma} N^{1 / 2-\sigma} \tau^{1 / 2} \Psi^{*}\left(N / p_{\tau}^{2}, p_{\tau}\right)^{1 / 2} .
\end{aligned}
$$

Estimates of $\ell_{1}$-type. The reader familiar with evaluation of Rademacher processes may wonder whether the brutal $\ell_{1}$-estimates

$$
\mathbb{E} \sup _{t \in \mathbb{R}}\left|\sum_{n \in \mathcal{E}_{\tau}} \varepsilon_{n} n^{-\sigma-i t}\right| \leq \sum_{n \in \mathcal{E}_{\tau}} n^{-\sigma}=: L(N, \tau)
$$

are useful at least in some zone of parameters. Indeed, for certain systems of random variables the $\ell_{1}$-estimates prove to give optimal order. However, 
in our context they are not useful. Actually, one can show that

$$
L(N, \tau) \geq c N^{1-\sigma} \Psi^{*}\left(N, p_{\tau}\right) \sim c N^{1-\sigma} \varrho\left(\frac{\log N}{\log p_{\tau}}\right) .
$$

This is too much for good upper bounds, as one can see from the following two examples. The first one handles large $\tau$ and the second one deals with small $\tau$.

1) Let $\tau \sim N^{h}$ with $1 / 2<h \leq 1$. Then we see that

$$
L(N, \tau) \geq c(h) N^{1-\sigma},
$$

while the upper bound from Theorem 1.1 yields a better estimate

$$
\mathbb{E} \sup _{t \in \mathbb{R}}\left|\sum_{n \in \mathcal{E}_{\tau}} \varepsilon_{n} n^{-\sigma-i t}\right| \leq C_{\sigma} \frac{N^{1 / 2-\sigma} \tau^{1 / 2}}{(\log N)^{1 / 2}} \approx C_{\sigma} \frac{N^{(1+h) / 2-\sigma}}{\log N} .
$$

The gap between the two upper bounds is at least logarithmic for $h=1$ and polynomial for $h<1$.

2) Let $\tau \sim \exp \left\{(\log \log N)^{A}\right\}$ with $A \geq 2$. Then we see that

$$
L(N, \tau) \geq c N^{1-\sigma} \varrho\left(\frac{\log N}{(\log \log N)^{A}}\right) \geq c N^{1-\sigma} \exp \left(\frac{-c \log N}{(\log \log N)^{A-1}}\right),
$$

while the upper bound from Theorem 1.2 yields a better estimate

$$
\mathbb{E} \sup _{t \in \mathbb{R}}\left|\sum_{n \in \mathcal{E}_{\tau}} \varepsilon_{n} n^{-\sigma-i t}\right| \leq C_{\sigma} N^{1 / 2-\sigma} \exp \left(\frac{-c \log N}{(\log \log N)^{A-1}}\right) .
$$

The gap between the two upper bounds is polynomial. One observes that the $\ell_{1}$-estimate becomes even worse when $\tau$ decreases and approaches the critical zone.

Rudin-Shapiro polynomials. The upper bound in Theorem 1.2 is known to be related to the Rudin-Shapiro problem for Dirichlet polynomials. Let us recall first the classical setting. For any trigonometric polynomial we have

$$
\frac{\sum_{n=0}^{N-1}\left|a_{n}\right|}{\sqrt{N}} \leq \sup _{t \in \mathbb{R}}\left|\sum_{n=0}^{N-1} a_{n} e^{i n t}\right| \leq \sum_{n=0}^{N-1}\left|a_{n}\right| .
$$

To get the lower bound one applies the inequality between the sup-norm and $L_{2}$-norm, the orthogonality of $\left(e^{i n t}\right)_{n}$ and the Hölder inequality.

Rudin and Shapiro constructed a fairly simple sequence $a_{n} \in\{-1,+1\}$ such that the right order of the lower bound is attained:

$$
\sup _{t \in \mathbb{R}}\left|\sum_{n=0}^{N-1} a_{n} e^{i n t}\right| \leq(2+\sqrt{2}) \sqrt{N+1} \sim(2+\sqrt{2}) \frac{\sum_{n=0}^{N-1}\left|a_{n}\right|}{\sqrt{N}} .
$$


Consider now Dirichlet polynomials instead of trigonometric ones. It is known from $[\mathrm{KQ}]$ and $[\mathrm{Q} 3]$ that for any $\left(a_{n}\right)$,

$$
\sup _{t \in \mathbb{R}}\left|\sum_{n=0}^{N-1} a_{n} n^{i t}\right| \geq \alpha_{1} \frac{\sum_{n=0}^{N-1}\left|a_{n}\right|}{\sqrt{N}} \exp \left\{\beta_{1} \sqrt{\log N \log \log N}\right\},
$$

and for some $\left(a_{n}\right)$,

$$
\sup _{t \in \mathbb{R}}\left|\sum_{n=0}^{N-1} a_{n} n^{i t}\right| \leq \alpha_{2} \frac{\sum_{n=0}^{N-1}\left|a_{n}\right|}{\sqrt{N}} \exp \left\{\beta_{2} \sqrt{\log N \log \log N}\right\},
$$

with some universal constants $\alpha_{1,2}, \beta_{1,2}$.

Therefore, the lower bound for Dirichlet polynomials is necessarily worse than in the classical case. Notice also that the construction of example (1.7) in [Q3] is a probabilistic one; no explicit example of Rudin-Shapiro type is known for Dirichlet polynomials. It turns out that Theorem 1.2 generates a new family of random polynomials satisfying (1.7).

Indeed, take any $\sigma \in[0,1 / 2)$ and choose $\tau$ in the optimal way. Namely, let

$$
\log \tau \sim\left(\frac{\log N}{2}\right)^{1 / 2}(\log \log N)^{1 / 2} .
$$

Set $a_{n}=\varepsilon_{n} n^{-\sigma} 1_{\left\{n \in \mathcal{E}_{\tau}\right\}}$. It is easy to see that

$$
\frac{\sum_{n=0}^{N}\left|a_{n}\right|}{\sqrt{N}}=\frac{\sum_{n \in \mathcal{E}_{\tau}} n^{-\sigma}}{\sqrt{N}} \geq c N^{1 / 2-\sigma} \Psi_{*}\left(N, p_{\tau}\right),
$$

while by Theorem 1.2 we have the bound for the average of the left hand side in (1.7):

$$
\begin{aligned}
\mathbb{E} \sup _{t \in \mathbb{R}} & \left|\sum_{n=0}^{N-1} a_{n} n^{i t}\right| \leq C_{\sigma} N^{1 / 2-\sigma} \tau^{1 / 2} \Psi^{*}\left(\frac{N}{p_{\tau}^{2}}, p_{\tau}\right)^{1 / 2} \\
& =C_{\sigma} N^{1 / 2-\sigma} \exp \left\{\frac{1}{2}\left(\frac{\log N}{2}\right)^{1 / 2}(\log \log N)^{1 / 2}+\frac{1}{2} \log \Psi^{*}\left(\frac{N}{p_{\tau}^{2}}, p_{\tau}\right)\right\} .
\end{aligned}
$$

Since by the properties of the Dickman function,

$$
\begin{aligned}
\log \Psi^{*}\left(\frac{N}{p_{\tau}^{2}}, p_{\tau}\right) & \sim \log \varrho\left(\frac{\log \left(N / p_{\tau}\right)}{\log p_{\tau}}\right) \sim-\frac{\log \left(N / p_{\tau}\right)}{\log p_{\tau}} \log \frac{\log \left(N / p_{\tau}\right)}{\log p_{\tau}} \\
& \sim-\frac{\log N}{\log \tau} \log \frac{\log N}{\log \tau} \sim-(2 \log N)^{1 / 2} \frac{(\log \log N)^{1 / 2}}{2} \\
& =-\left(\frac{\log N}{2}\right)^{1 / 2}(\log \log N)^{1 / 2},
\end{aligned}
$$


and by the same arguments

$$
\log \Psi^{*}\left(N, p_{\tau}\right) \sim-\left(\frac{\log N}{2}\right)^{1 / 2}(\log \log N)^{1 / 2},
$$

we finally obtain

$$
\mathbb{E} \sup _{t \in \mathbb{R}}\left|\sum_{n=0}^{N-1} a_{n} n^{i t}\right| \leq \frac{C_{\sigma}}{c} \frac{\sum_{n=0}^{N}\left|a_{n}\right|}{\sqrt{N}} \exp \left\{\left(\frac{\log N}{2}\right)^{1 / 2}(\log \log N)^{1 / 2}\right\},
$$

as required in (1.7).

A particular case of this example with $\sigma=0$ was considered in [Q3]. Our calculation yields a slightly better constant in the exponent. The question about the best possible constant raised in $[\mathrm{KQ}]$ seems still to be open.

2. Proof of the upper bound in Theorem 1.1. The principle of the proof of the upper bound is as follows. Once we reduce to the study of a random polynomial $Q$ on the multidimensional torus by using (1.1), the proof consists of two different steps based on a decomposition $Q=Q_{1}+Q_{2}$. The study of the supremum of the polynomial $Q_{1}$ is made by using the metric entropy method.

The supremum of $Q_{2}$ is handled by using first the contraction principle, reducing the study to the one of a complex-valued Gaussian process. The latter task is carried out by applying Slepian's comparison lemma, and by a careful study of the $L^{2}$-metric induced by this process.

Now, we turn to the rigorous proof of the upper bound and introduce some notation.

We can represent $\mathcal{E}_{\tau}$ as the union of disjoint sets

$$
E_{j}=\left\{2 \leq n \leq N: P^{+}(n)=p_{j}\right\}, \quad j=1, \ldots, \tau .
$$

For $\underline{z} \in \mathbb{T}^{\tau}$ we put

$$
Q(\underline{z})=\sum_{j=1}^{\tau} \sum_{n \in E_{j}} \varepsilon_{n} n^{-\sigma} e^{2 i \pi\langle\underline{a}(n), \underline{z}\rangle} .
$$

By (1.1) we have

$$
\sup _{t \in \mathbb{R}}\left|\sum_{j=1}^{\tau} \sum_{n \in E_{j}} \varepsilon_{n} n^{-\sigma-i t}\right|=\sup _{\underline{z} \in \mathbb{T}^{\tau}}|Q(\underline{z})| .
$$

Let $1 \leq \nu<\tau$ be fixed. Write $Q=Q_{1}+Q_{2}$ where

$$
\begin{aligned}
Q_{1}(\underline{z}) & =\sum_{P^{+}(n) \leq p_{\nu}} \varepsilon_{n} n^{-\sigma} e^{2 i \pi\langle\underline{a}(n), \underline{z}\rangle}, \\
Q_{2}(\underline{z}) & =\sum_{p_{\nu}<P^{+}(n) \leq p_{\tau}} \varepsilon_{n} n^{-\sigma} e^{2 i \pi\langle\underline{a}(n), \underline{z}\rangle} .
\end{aligned}
$$


First, we estimate the supremum of $Q_{2}$. We introduce the random process

$$
X^{\varepsilon}(\gamma)=\sum_{\nu<j \leq \tau} \alpha_{j} \sum_{n \in E_{j}} \varepsilon_{n} n^{-\sigma} \beta_{n / p_{j}}, \quad \gamma \in \Gamma,
$$

where $\gamma=\left(\left(\alpha_{j}\right)_{\nu<j \leq \tau},\left(\beta_{m}\right)_{1 \leq m \leq N / 2}\right)$ and $\Gamma=\left\{\gamma:\left|\alpha_{j}\right| \vee\left|\beta_{m}\right| \leq 1\right.$, $\nu<j \leq \tau, 1 \leq m \leq N / 2\}$. Writing

$$
\begin{aligned}
Q_{2}(\underline{z}) & =\sum_{\nu<j \leq \tau} e^{2 i \pi z_{j}} \sum_{n \in E_{j}} \varepsilon_{n} n^{-\sigma} e^{2 i \pi\left\{\sum_{k \neq j} a_{k}(n) z_{k}+\left[a_{j}(n)-1\right] z_{j}\right\}} \\
& =\sum_{\nu<j \leq \tau} e^{2 i \pi z_{j}} \sum_{n \in E_{j}} \varepsilon_{n} n^{-\sigma} e^{2 i \pi\left\{\sum_{k} a_{k}\left(n / p_{j}\right) z_{k}\right\}}
\end{aligned}
$$

and considering separately the imaginary and real parts of the exponents, it follows that $Q_{2}(\underline{z})$ can be written as the sum of four terms, each being of the form

$$
\eta \sum_{\nu<j \leq \tau} \alpha_{j} \sum_{n \in E_{j}} \varepsilon_{n} n^{-\sigma} \beta_{n / p_{j}}
$$

where $\eta \in\{1, i,-i,-1\}$, and

$$
\begin{aligned}
\alpha_{j} & =\left\{\begin{array}{l}
\cos \left(2 \pi z_{j}\right), \\
\operatorname{or} \\
\sin \left(2 \pi z_{j}\right),
\end{array}\right. \\
\beta_{m} & =\left\{\begin{array}{l}
\cos \left(2 \pi \sum_{k} a_{k}(m) z_{k}\right), \\
\text { or } \quad 1 \leq m \leq N / 2 . \\
\sin \left(2 \pi \sum_{k} a_{k}(m) z_{k}\right),
\end{array}\right.
\end{aligned}
$$

Therefore, we obtain

$$
\sup _{\underline{z} \in \mathbb{T}^{\tau}}\left|Q_{2}(\underline{z})\right| \leq 4 \sup _{\gamma \in \Gamma}\left|X^{\varepsilon}(\gamma)\right| .
$$

By the contraction principle ([K, pp. 16-17])

$$
\mathbb{E} \sup _{\underline{z} \in \mathbb{T}^{\tau}}\left|Q_{2}(\underline{z})\right| \leq 4 \sqrt{\pi / 2} \mathbb{E} \sup _{\gamma \in \Gamma}|X(\gamma)|
$$

where $\{X(\gamma), \gamma \in \Gamma\}$ is the same process as $X^{\varepsilon}(\gamma)$ except that the Rademacher random variables $\varepsilon_{n}$ are replaced by independent $\mathcal{N}(0,1)$ random variables $\mu_{n}$ :

$$
X(\gamma)=\sum_{\nu<j \leq \tau} \alpha_{j} \sum_{n \in E_{j}} \mu_{n} n^{-\sigma} \beta_{n / p_{j}} .
$$

The problem now reduces to estimating the supremum of the real-valued 
Gaussian process $X$. To this end, we examine the $L^{2}$-norm of its increments:

$$
\begin{aligned}
\left\|X_{\gamma}-X_{\gamma^{\prime}}\right\|_{2}^{2} & =\sum_{\nu<j \leq \tau} \sum_{n \in E_{j}} n^{-2 \sigma}\left[\alpha_{j} \beta_{n / p_{j}}-\alpha_{j}^{\prime} \beta_{n / p_{j}}^{\prime}\right]^{2} \\
& \leq 2 \sum_{\nu<j \leq \tau} \sum_{n \in E_{j}} n^{-2 \sigma}\left[\left(\alpha_{j}-\alpha_{j}^{\prime}\right)^{2}+\left(\beta_{n / p_{j}}-\beta_{n / p_{j}}^{\prime}\right)^{2}\right],
\end{aligned}
$$

where we have used the identity $\alpha_{j} \beta_{n / p_{j}}-\alpha_{j}^{\prime} \beta_{n / p_{j}}^{\prime}=\left(\alpha_{j}-\alpha_{j}^{\prime}\right) \beta_{n / p_{j}}+$ $\left(\beta_{n / p_{j}}-\beta_{n / p_{j}}^{\prime}\right) \alpha_{j}^{\prime}$.

The " $\alpha$ " part is easily controlled as follows:

$$
\begin{aligned}
\sum_{\nu<j \leq \tau} \sum_{n \in E_{j}} n^{-2 \sigma}\left(\alpha_{j}-\alpha_{j}^{\prime}\right)^{2} & \leq \sum_{\nu<j \leq \tau}\left(\alpha_{j}-\alpha_{j}^{\prime}\right)^{2} p_{j}^{-2 \sigma} \sum_{m \leq N / p_{j}} m^{-2 \sigma} \\
& \leq C_{\sigma} \sum_{\nu<j \leq \tau}\left(\alpha_{j}-\alpha_{j}^{\prime}\right)^{2}\left(\frac{N^{1-2 \sigma}}{p_{j}}\right) .
\end{aligned}
$$

For the " $\beta$ " part, we have

$$
\begin{aligned}
\sum_{\nu<j \leq \tau} \sum_{n \in E_{j}} \frac{\left(\beta_{n / p_{j}}-\beta_{n / p_{j}}^{\prime}\right)^{2}}{n^{2 \sigma}} & \leq \sum_{m \leq N / p_{\nu}}\left(\beta_{m}-\beta_{m}^{\prime}\right)^{2}\left(\sum_{\substack{\nu<j \leq \tau \\
m p_{j} \leq N}} \frac{1}{\left(m p_{j}\right)^{2 \sigma}}\right) \\
& =: \sum_{m \leq N / p_{\nu}} K_{m}^{2}\left(\beta_{m}-\beta_{m}^{\prime}\right)^{2} .
\end{aligned}
$$

Now we estimate the coefficients $K_{m}$. Consider two cases.

1) $m \leq N / p_{\tau}$. Then $m p_{j} \leq m p_{\tau} \leq N$ for all $j \leq \tau$ and, by using the standard estimate (see [HW, Theorem 8, p. 10])

$$
p_{j} \sim j \log j,
$$

we have

$$
\begin{aligned}
K_{m}^{2} & =\sum_{\nu<j \leq \tau}\left(m p_{j}\right)^{-2 \sigma} \leq m^{-2 \sigma} \sum_{j \leq \tau} p_{j}^{-2 \sigma} \\
& \leq C m^{-2 \sigma} \sum_{j \leq \tau}(j \log j)^{-2 \sigma}=C_{\sigma} m^{-2 \sigma} \tau^{1-2 \sigma}(\log \tau)^{-2 \sigma} \\
& \leq C_{\sigma} m^{-2 \sigma} \frac{\tau}{p_{\tau}^{2 \sigma}} .
\end{aligned}
$$

Thus

$$
\begin{aligned}
\sum_{m \leq N / p_{\tau}} K_{m} & \leq C_{\sigma} \frac{\tau^{1 / 2}}{p_{\tau}^{\sigma}} \sum_{m \leq N / p_{\tau}} m^{-\sigma} \leq C_{\sigma}\left(\frac{N}{p_{\tau}}\right)^{1-\sigma} \frac{\tau^{1 / 2}}{p_{\tau}^{\sigma}} \\
& =\frac{C_{\sigma} N^{1-\sigma} \tau^{1 / 2}}{p_{\tau}} \leq \frac{C_{\sigma} N^{1-\sigma}}{\tau^{1 / 2} \log \tau} .
\end{aligned}
$$


2) $N / p_{\nu} \geq m>N / p_{\tau}$. Then take a unique $k \in(\nu, \tau]$ such that $N / p_{k}<$ $m \leq N / p_{k-1}$. We have

$$
\begin{aligned}
K_{m}^{2} & =\sum_{\nu<j \leq k-1}\left(m p_{j}\right)^{-2 \sigma} \leq m^{-2 \sigma} \sum_{j \leq k-1} p_{j}^{-2 \sigma} \\
& \leq C_{\sigma} m^{-2 \sigma} \sum_{j \leq k}(j \log j)^{-2 \sigma} \leq C_{\sigma} m^{-2 \sigma} \frac{k^{1-2 \sigma}}{(\log k)^{2 \sigma}} \\
& \leq C_{\sigma} m^{-2 \sigma} \frac{k}{p_{k}^{2 \sigma}} \leq C_{\sigma} m^{-2 \sigma} \frac{k}{(N / m)^{2 \sigma}} \\
& =C_{\sigma} \frac{k}{N^{2 \sigma}} .
\end{aligned}
$$

Since $k \log k \leq C p_{k} \leq C N / m$, we have

$$
k \leq C \frac{N}{m}\left(\log \left(\frac{N}{m}\right)\right)^{-1} .
$$

We arrive at $K_{m} \leq C_{\sigma} N^{-\sigma}(N / m)^{1 / 2}(\log (N / m))^{-1 / 2}$. It follows that

$$
\begin{aligned}
\sum_{m \leq N / p_{\nu}} K_{m} & \leq C_{\sigma} N^{-\sigma} \sum_{m \leq N / p_{\nu}}(N / m)^{1 / 2}(\log (N / m))^{-1 / 2} \\
& \leq C_{\sigma} N^{1-\sigma} \int_{0}^{1 / p_{\nu}} u^{-1 / 2}(\log (1 / u))^{-1 / 2} d u \\
& \leq C_{\sigma} N^{1-\sigma} p_{\nu}^{-1 / 2}\left(\log p_{\nu}\right)^{-1 / 2} \leq \frac{C_{\sigma} N^{1-\sigma}}{\nu^{1 / 2} \log \nu}
\end{aligned}
$$

Now define a second Gaussian process by putting, for all $\gamma \in \Gamma$,

$$
Y(\gamma)=\sum_{\nu<j \leq \tau}\left(\frac{N^{1-2 \sigma}}{p_{j}}\right)^{1 / 2} \alpha_{j} \xi_{j}^{\prime}+\sum_{m \leq N / p_{\nu}} K_{m} \beta_{m} \xi_{m}^{\prime \prime}=: Y_{\gamma}^{\prime}+Y_{\gamma}^{\prime \prime},
$$

where $\xi_{i}^{\prime}, \xi_{j}^{\prime \prime}$ are independent $\mathcal{N}(0,1)$ random variables. It follows from $(2.1)$ and (2.2) that for some suitable constant $C_{\sigma}$, one has the comparison relations: for all $\gamma, \gamma^{\prime} \in \Gamma$,

$$
\left\|X_{\gamma}-X_{\gamma^{\prime}}\right\|_{2} \leq C_{\sigma}\left\|Y_{\gamma}-Y_{\gamma^{\prime}}\right\|_{2} .
$$

By virtue of the Slepian comparison lemma (see [L, Theorem 4, p. 190]), since $X_{0}=Y_{0}=0$, we have

$$
\mathbb{E} \sup _{\gamma \in \Gamma}\left|X_{\gamma}\right| \leq 2 \mathbb{E} \sup _{\gamma \in \Gamma} X_{\gamma} \leq 2 C_{\sigma} \mathbb{E} \sup _{\gamma \in \Gamma} Y_{\gamma} \leq 2 C_{\sigma} \mathbb{E} \sup _{\gamma \in \Gamma}\left|Y_{\gamma}\right|
$$

It remains to evaluate the supremum of $Y$. First of all,

$$
\mathbb{E} \sup _{\gamma \in \Gamma}\left|Y^{\prime}(\gamma)\right| \leq N^{1 / 2-\sigma} \sum_{\nu<j \leq \tau} p_{j}^{-1 / 2} .
$$


By (2.3), we have

$$
\sum_{\nu<j \leq \tau} p_{j}^{-1 / 2} \leq \sum_{1<j \leq \tau} p_{j}^{-1 / 2} \leq \frac{C \tau^{1 / 2}}{(\log \tau)^{1 / 2}},
$$

thus

$$
\mathbb{E} \sup _{\gamma \in \Gamma}\left|Y^{\prime}(\gamma)\right| \leq C N^{1 / 2-\sigma} \frac{\tau^{1 / 2}}{(\log \tau)^{1 / 2}} .
$$

To control the supremum of $Y^{\prime \prime}$, we use our estimates for the sums of $K_{m}$ to obtain

$$
\begin{aligned}
\mathbb{E} \sup _{\gamma \in \Gamma}\left|Y^{\prime \prime}(\gamma)\right| & \leq \sum_{m \leq N / p_{\nu}} K_{m} \\
& \leq C_{\sigma}\left(\frac{N^{1-\sigma}}{\nu^{1 / 2} \log \nu}+\frac{N^{1-\sigma}}{\tau^{1 / 2} \log \tau}\right) \leq \frac{C_{\sigma} N^{1-\sigma}}{\nu^{1 / 2} \log \nu} .
\end{aligned}
$$

Now, we turn to the supremum of $Q_{1}$. To this end, we introduce the auxiliary Gaussian process

$$
\Upsilon(\underline{z})=\sum_{P^{+}(n) \leq p_{\nu}} n^{-\sigma}\left\{\vartheta_{n} \cos 2 \pi\langle\underline{a}(n), \underline{z}\rangle+\vartheta_{n}^{\prime} \sin 2 \pi\langle\underline{a}(n), \underline{z}\rangle\right\}, \quad \underline{z} \in \mathbb{T}^{\nu},
$$

where $\vartheta_{i}, \vartheta_{j}^{\prime}$ are independent $\mathcal{N}(0,1)$ random variables. By symmetrization (see e.g. Lemma 2.3, p. 269 in [PSW]),

$$
\mathbb{E} \sup _{\underline{z} \in \mathbb{T}^{\nu}}\left|Q_{1}(\underline{z})\right| \leq \sqrt{8 \pi} \mathbb{E} \sup _{\underline{z} \in \mathbb{T}^{\nu}}|\Upsilon(\underline{z})|,
$$

so that we are again led to evaluating the supremum of a real-valued Gaussian process. For $\underline{z}, \underline{z}^{\prime} \in \mathbb{T}^{\nu}$ put $\|\Upsilon(\underline{z})-\Upsilon(\underline{z})\|_{2}=: d\left(\underline{z}, \underline{z}^{\prime}\right)$, and observe that

$$
\begin{aligned}
& d\left(\underline{z}, \underline{z}^{\prime}\right)^{2}=4 \sum_{n: P^{+}(n) \leq p_{\nu}} \frac{1}{n^{2 \sigma}} \sin ^{2}\left(\pi\left\langle\underline{a}(n), \underline{z}-\underline{z}^{\prime}\right\rangle\right) \\
& \quad \leq 4 \pi^{2} \sum_{n: P^{+}(n) \leq p_{\nu}} \frac{1}{n^{2 \sigma}}\left|\left\langle\underline{a}(n), \underline{z}-\underline{z}^{\prime}\right\rangle\right|^{2} \\
& \quad \leq 4 \pi^{2} \sum_{n: P^{+}(n) \leq p_{\nu}} n^{-2 \sigma}\left[\sum_{j=1}^{\nu} a_{j}(n)\left|z_{j}-z_{j}^{\prime}\right|\right]^{2} \\
& =4 \pi^{2} \sum_{n: P^{+}(n) \leq p_{\nu}} \sum_{j_{1}, j_{2}=1}^{\nu} a_{j_{1}}(n) a_{j_{2}}(n)\left|z_{j_{1}}-z_{j_{1}}^{\prime}\right|\left|z_{j_{2}}-z_{j_{2}}^{\prime}\right| n^{-2 \sigma}
\end{aligned}
$$




$$
\begin{aligned}
& =4 \pi^{2} \sum_{j_{1}, j_{2}=1}^{\nu} \sum_{n: P^{+}(n) \leq p_{\nu}} a_{j_{1}}(n) a_{j_{2}}(n)\left|z_{j_{1}}-z_{j_{1}}^{\prime}\right|\left|z_{j_{2}}-z_{j_{2}}^{\prime}\right| n^{-2 \sigma} \\
& \leq 4 \pi^{2} \sum_{j_{1}, j_{2}=1}^{\nu}\left|z_{j_{1}}-z_{j_{1}}^{\prime}\right|\left|z_{j_{2}}-z_{j_{2}}^{\prime}\right| \sum_{b_{1}, b_{2}=1}^{\infty} b_{1} b_{2} \sum_{n \leq N, a_{j_{1}}(n)=b_{1}, a_{j_{2}}(n)=b_{2}} n^{-2 \sigma} \\
& \leq 4 \pi^{2} \sum_{j_{1}, j_{2}=1}^{\nu}\left|z_{j_{1}}-z_{j_{1}}^{\prime}\right|\left|z_{j_{2}}-z_{j_{2}}^{\prime}\right| \sum_{b_{1}, b_{2}=1}^{\infty} b_{1} b_{2} p_{j_{1}}^{-2 b_{1} \sigma} p_{j_{2}}^{-2 b_{2} \sigma} \sum_{k \leq N p_{j_{1}}^{-b_{1}} p_{j_{2}}^{-b_{2}}} k^{-2 \sigma}{ }_{P_{(k)} \leq p_{\nu}} \sum_{j_{1}, j_{2}=1}^{\nu}\left|z_{j_{1}}-z_{j_{1}}^{\prime}\right|\left|z_{j_{2}}-z_{j_{2}}^{\prime}\right| \sum_{b_{1}, b_{2}=1}^{\infty} b_{1} b_{2} p_{j_{1}}^{-2 b_{1} \sigma} p_{j_{2}-2 b_{2} \sigma} p_{\left.p_{j_{1}}^{-b_{1}} p_{j_{2}}^{-b_{2}}\right]^{1-2 \sigma}} \\
& \leq C_{\sigma} N^{1-2 \sigma} \sum_{\sum_{1}, j_{2}=1}^{\nu}\left|z_{j_{1}}-z_{j_{1}}^{\prime}\right|\left|z_{j_{2}}-z_{j_{2}}^{\prime}\right| \sum_{b_{1}, b_{2}=1}^{\infty} b_{1} b_{2} p_{j_{1}}^{-b_{1}} p_{j_{2}}^{-b_{2}} \\
& \left.=C_{\sigma} N^{1-2 \sigma} \sum_{j=1}^{\nu}\left|z_{j}-z_{j}^{\prime}\right| \sum_{b=1}^{\infty} b p_{j}^{-b}\right\}^{2} .
\end{aligned}
$$

Thus,

$$
d\left(z, z^{\prime}\right) \leq C_{\sigma} N^{1 / 2-\sigma}\left\{\sum_{j=1}^{\nu}\left|z_{j}-z_{j}^{\prime}\right| \sum_{b=1}^{\infty} b p_{j}^{-b}\right\}
$$

REMARK. In the middle of the long calculation, we did not use the fact that the variable $k$ satisfies $P^{+}(k) \leq p_{\nu}$. Actually, this observation permits introducing an extra factor connected with the Dickman function, something like $\varrho(\log N / \log \nu)$. This is helpful once $\nu$ is very small with respect to $N$ (see the upper bound in Theorem 1.2).

Now we explore the entropy properties of the metric space $\left(\mathbb{T}^{\nu}, d\right)$. To do this, take $\varepsilon \in(0,1)$ and cover $\mathbb{T}^{\nu}$ by rectangular cells so that if $z$ and $z^{\prime}$ belong to the same cell, then

$$
\left|z_{j}-z_{j}^{\prime}\right| \leq \begin{cases}\varepsilon / \log \log \nu, & 1 \leq j \leq \nu^{1 / 2} \\ \varepsilon, & \nu^{1 / 2}<j \leq \nu\end{cases}
$$

Thus, every cell is a product of two cubes of different size and dimension. The necessary number of cells, $M(\varepsilon)$, is bounded as follows:

$$
M(\varepsilon) \leq\left(\frac{\log \log \nu}{\varepsilon}\right)^{\left[\nu^{1 / 2}\right]} \varepsilon^{-\left(\nu-\left[\nu^{1 / 2}\right]\right)}=(1 / \varepsilon)^{\nu}(\log \log \nu)^{\left[\nu^{1 / 2}\right]} .
$$

Let us now estimate the distance $d\left(z, z^{\prime}\right)$ for $z, z^{\prime}$ satisfying (2.8). By (2.7) 
we have

$$
d\left(z, z^{\prime}\right) \leq C_{\sigma} N^{1 / 2-\sigma}\left\{d_{1}+d_{2}+d_{3}\right\}
$$

where

$$
\begin{aligned}
& d_{1}=\sum_{j=1}^{\nu}\left|z_{j}-z_{j}^{\prime}\right| \sum_{b=2}^{\infty} b p_{j}^{-b}, \\
& d_{2}=\sum_{\nu^{1 / 2}<j \leq \nu}\left|z_{j}-z_{j}^{\prime}\right| p_{j}^{-1}, \\
& d_{3}=\sum_{j \leq \nu^{1 / 2}}\left|z_{j}-z_{j}^{\prime}\right| p_{j}^{-1} .
\end{aligned}
$$

For any $j \geq 1$ we have

$$
\sum_{b=2}^{\infty} b p_{j}^{-b}=\sum_{b=2}^{\infty} b\left(\frac{2}{p_{j}}\right)^{b} 2^{-b} \leq\left(\frac{2}{p_{j}}\right)^{2} \sum_{b=2}^{\infty} b 2^{-b}=C p_{j}^{-2} .
$$

Hence,

$$
d_{1} \leq\left(\sum_{j=1}^{\nu} C p_{j}^{-2}\right) \max _{j \leq \nu}\left|z_{j}-z_{j}^{\prime}\right| \leq C \varepsilon
$$

Similarly,

$$
\begin{aligned}
d_{2} & \leq\left(\sum_{\nu^{1 / 2}<j \leq \nu} p_{j}^{-1}\right) \max _{\nu^{1 / 2}<j \leq \nu}\left|z_{j}-z_{j}^{\prime}\right| \leq C\left(\sum_{\nu^{1 / 2}<j \leq \nu}(j \log j)^{-1}\right) \varepsilon \\
& \leq C \int_{\nu^{1 / 2}}^{\nu} \frac{d u}{u \log u} \varepsilon=C\left(\log \log \nu-\log \left(\frac{\log \nu}{2}\right)\right) \varepsilon=C(\log 2) \varepsilon .
\end{aligned}
$$

Finally,

$$
d_{3} \leq\left(\sum_{j=1}^{\nu} p_{j}^{-1}\right) \max _{j \leq \nu^{1 / 2}}\left|z_{j}-z_{j}^{\prime}\right| \leq C\left(\sum_{j=1}^{\nu}(j \log j)^{-1}\right) \frac{\varepsilon}{\log \log \nu} \leq C \varepsilon .
$$

By summing up the three estimates, we have $d\left(z, z^{\prime}\right) \leq C_{\sigma} N^{1 / 2-\sigma} \varepsilon$, which enables the estimation of the metric entropy.

Let $\mathcal{N}\left(\mathbb{T}^{\nu}, d, u\right)$ be the minimal number of balls of radius $u$ that cover the space $\left(\mathbb{T}^{\nu}, d\right)$. We have

$$
\log \mathcal{N}\left(\mathbb{T}^{\nu}, d, C_{\sigma} N^{1 / 2-\sigma} \varepsilon\right) \leq \log M(\varepsilon) \leq \nu|\log \varepsilon|+\nu^{1 / 2} \cdot \log \log \log \nu .
$$

Observe also that

$$
\|\Upsilon(\underline{z})\|_{2} \leq C_{\sigma} N^{1 / 2-\sigma}, \quad \underline{z} \in \mathbb{T}^{\nu}
$$

Hence, $D:=\operatorname{diam}\left(\mathbb{T}^{\nu}, d\right) \leq C_{\sigma} N^{1 / 2-\sigma}$, and by the classical Dudley entropy 
theorem (see [L, Theorem 1, p. 179]), for any fixed $\underline{z} \in \mathbb{T}^{\nu}$,

$$
\begin{aligned}
\mathbb{E} \sup _{\underline{z^{\prime}} \in \mathbb{T}^{\nu}}\left|\Upsilon\left(\underline{z}^{\prime}\right)-\Upsilon(\underline{z})\right| & \leq C_{\sigma} \int_{0}^{D}\left[\log \mathcal{N}\left(\mathbb{T}^{\nu}, d, u\right)\right]^{1 / 2} d u \\
& \leq C_{\sigma} \int_{0}^{C_{\sigma} N^{1 / 2-\sigma}}\left[\log \mathcal{N}\left(\mathbb{T}^{\nu}, d, u\right)\right]^{1 / 2} d u \\
& =C_{\sigma} N^{1 / 2-\sigma} \int_{0}^{1}\left[\log \mathcal{N}\left(\mathbb{T}^{\nu}, d, C_{\sigma} N^{1 / 2-\sigma} \varepsilon\right)\right]^{1 / 2} d \varepsilon \\
& \leq C_{\sigma} N^{1 / 2-\sigma} \int_{0}^{1}\left[\nu|\log \varepsilon|+\log \log \log \nu \cdot \nu^{1 / 2}\right]^{1 / 2} d \varepsilon \\
& \leq C_{\sigma} N^{1 / 2-\sigma} \nu^{1 / 2}
\end{aligned}
$$

Using again (2.10), we have

$$
\mathbb{E} \sup _{\underline{z}^{\prime} \in \mathbb{T}^{\nu}}\left|\Upsilon\left(\underline{z}^{\prime}\right)\right| \leq C_{\sigma} N^{1 / 2-\sigma} \nu^{1 / 2} .
$$

The final stage of the proof provides the optimal choice of the parameter $\nu$ balancing the quantities (2.4), (2.5), and (2.11). As the theorem's claim suggests, we consider three cases.

CASE 1: $N^{1 / 2} \leq \tau \leq N$. Obviously, this case contains the results of Halász and Queffélec. In this case we choose

$$
\nu=\frac{\tau}{\log N},
$$

thus balancing (2.4) and (2.11). We obtain from both terms the bound $C_{\sigma} N^{1 / 2-\sigma} \tau^{1 / 2} /(\log N)^{1 / 2}$, while the term (2.5) is negligible. The correctness condition $\nu \leq \tau$ is obvious.

CASE 2: $N^{1 / 2}(\log N)^{-1} \leq \tau \leq N^{1 / 2}$. In this case we choose

$$
\nu=N^{1 / 2}(\log N)^{-1},
$$

thus balancing (2.5) and (2.11). We obtain from both terms the bound $C_{\sigma} N^{3 / 4-\sigma} /(\log N)^{1 / 2}$, while the term (2.4) is negligible. The correctness condition $\nu \leq \tau$ is obvious for the range under consideration.

CASE 3: $1 \leq \tau \leq N^{1 / 2}(\log N)^{-1}$. Here we just set $\nu=\tau$. This means that we do not need the splitting of the polynomial in two parts. Formally, the quantities (2.4) and (2.5) are not necessary and we obtain the bound $C_{\sigma} N^{1 / 2-\sigma} \tau^{1 / 2}$ directly from (2.11).

The proof of the upper bound is complete. 
3. Proof of the lower bound in Theorem 1.1. Let $\underline{d}=\left\{d_{n}, n \geq 1\right\}$ be a sequence of reals. Recall that by (1.1) we have

$$
\sup _{t \in \mathbb{R}}\left|\sum_{j=1}^{\tau} \sum_{n \in E_{j}} d_{n} \varepsilon_{n} n^{-\sigma-i t}\right|=\sup _{\underline{z} \in \mathbb{T}^{\tau}}|Q(\underline{z})|,
$$

where

$$
Q(\underline{z})=\sum_{j=1}^{\tau} \sum_{n \in E_{j}} d_{n} \varepsilon_{n} n^{-\sigma} e^{2 i \pi\langle\underline{a}(n), \underline{z}\rangle} .
$$

Consider the subset $\mathcal{Z}$ of $\mathbb{T}^{\tau}$ defined by

$$
\begin{aligned}
\mathcal{Z}=\left\{\underline{z}=\left\{z_{j}, 1 \leq j \leq \tau\right\}: z_{j}=0\right. & \text { if } j \leq \tau / 2, \\
& \text { and } \left.z_{j} \in\{0,1 / 2\} \text { if } j \in(\tau / 2, \tau]\right\} .
\end{aligned}
$$

Observe that the imaginary part of $Q$ vanishes on $\mathcal{Z}$, since for any $\underline{z} \in \mathcal{Z}$ and any $n$,

$$
e^{2 i \pi\langle\underline{a}(n), \underline{z}\rangle}=\cos (2 \pi\langle\underline{a}(n), \underline{z}\rangle)=(-1)^{2\langle\underline{a}(n), \underline{z}\rangle} .
$$

Hence, $Q$ takes the following simple form on $\mathcal{Z}$ :

$$
Q(\underline{z})=\sum_{\tau / 2<j \leq \tau} \sum_{n \in E_{j}} d_{n} \varepsilon_{n} n^{-\sigma}(-1)^{2\langle\underline{a}(n), \underline{z}\rangle} .
$$

This is no longer a trigonometric polynomial, but simply a finite rank Rademacher process.

For $j \in(\tau / 2, \tau]$ define

$$
\mathcal{L}_{j}=\left\{n=p_{j} \widetilde{n}: \widetilde{n} \leq N / p_{j} \text { and } P^{+}(\widetilde{n}) \leq p_{\tau / 2}\right\} .
$$

Since $E_{j} \supset \mathcal{L}_{j}, j=1, \ldots, \tau$, the sets $\mathcal{L}_{j}$ are pairwise disjoint. For $\underline{z} \in \mathcal{Z}$ put

$$
Q^{\prime}(\underline{z})=\sum_{\tau / 2<j \leq \tau} \sum_{n \in \mathcal{L}_{j}} d_{n} \varepsilon_{n} n^{-\sigma}(-1)^{2\langle\underline{a}(n), \underline{z}\rangle} .
$$

We now recall a useful fact.

Lemma 3.1. Let $X=\left\{X_{z}, z \in Z\right\}$ and $Y=\left\{Y_{z}, z \in Z\right\}$ be two finite sets of random variables defined on a common probability space. Assume that $X$ and $Y$ are independent and that the random variables $Y_{z}$ are all centered. Then

$$
\mathbb{E} \sup _{z \in Z}\left|X_{z}+Y_{z}\right| \geq \mathbb{E} \sup _{z \in Z}\left|X_{z}\right| .
$$

Proof. Let $\mathcal{F}$ be the $\sigma$-field generated by $Y$. Then

$$
\begin{aligned}
\mathbb{E} \sup _{z \in Z}\left|X_{z}+Y_{z}\right| & =\mathbb{E}\left[\mathbb{E}\left(\sup _{z \in Z}\left|X_{z}+Y_{z}\right| \mid \mathcal{F}\right)\right] \geq \mathbb{E}\left[\sup _{z \in Z}\left|\mathbb{E}\left(X_{z}+Y_{z} \mid \mathcal{F}\right)\right|\right] \\
& =\mathbb{E}\left(\sup _{z \in Z}\left|X_{z}+\mathbb{E} Y_{z}\right|\right)=\mathbb{E} \sup _{z \in Z}\left|X_{z}\right| .
\end{aligned}
$$


Clearly, since $\left\{Q(\underline{z})-Q^{\prime}(\underline{z}), \underline{z} \in \mathcal{Z}\right\}$ and $\left\{Q^{\prime}(\underline{z}), \underline{z} \in \mathcal{Z}\right\}$ are independent, $\mathbb{E} \sup _{\underline{z} \in \mathcal{Z}}|Q(\underline{z})| \geq \mathbb{E} \sup _{\underline{z} \in \mathcal{Z}}\left|Q^{\prime}(\underline{z})\right|$.

We now proceed to a direct estimation of $Q^{\prime}(\underline{z})$ by proving

Proposition 3.2. There exists a universal constant $c$ such that for any system of coefficients $\left(d_{n}\right)$,

$$
c \sum_{\tau / 2<j \leq \tau}\left|\sum_{n \in \mathcal{L}_{j}} d_{n}^{2}\right|^{1 / 2} \leq \mathbb{E} \sup _{\underline{z} \in \mathcal{Z}}\left|Q^{\prime}(\underline{z})\right| \leq \sum_{\tau / 2<j \leq \tau}\left|\sum_{n \in \mathcal{L}_{j}} d_{n}^{2}\right|^{1 / 2} .
$$

Proof. For any $n \in \mathcal{L}_{j}$, we have $2\langle\underline{a}(n), \underline{z}\rangle=2 z_{j}$, so that

$$
\sum_{n \in \mathcal{L}_{j}} d_{n} \varepsilon_{n}(-1)^{2\langle\underline{a}(n), \underline{z}\rangle}=(-1)^{2 z_{j}} \sum_{n \in \mathcal{L}_{j}} d_{n} \varepsilon_{n}(\omega) .
$$

Thus

$$
Q^{\prime}(\underline{z})=\sum_{\tau / 2<j \leq \tau}(-1)^{2 z_{j}} \sum_{n \in \mathcal{L}_{j}} d_{n} \varepsilon_{n}(\omega) .
$$

Let $\omega \in \Omega$. We can select $z_{j}=z_{j}(\omega)=0$ or $1 / 2, \tau / 2<j \leq \tau$, according to the + or $-\operatorname{sign}$ of the sum $\sum_{n \in \mathcal{L}_{j}} d_{n} \varepsilon_{n}(\omega) n^{-\sigma}$. This implies that

$$
\sup _{\underline{z} \in \mathcal{Z}}\left|Q^{\prime}(\underline{z})\right|=\sum_{\tau / 2<j \leq \tau}\left|\sum_{n \in \mathcal{L}_{j}} d_{n} \varepsilon_{n}\right| .
$$

Consequently, by the Khinchin inequalities for Rademacher sums [KS],

$$
\begin{aligned}
\mathbb{E} \sup _{\underline{z} \in \mathcal{Z}}\left|Q^{\prime}(\underline{z})\right| & =\sum_{\tau / 2<j \leq \tau} \mathbb{E}\left|\sum_{n \in \mathcal{L}_{j}} d_{n} \varepsilon_{n}\right| \geq c \sum_{\tau / 2<j \leq \tau}\left(\mathbb{E}\left|\sum_{n \in \mathcal{L}_{j}} d_{n} \varepsilon_{n}\right|^{2}\right)^{1 / 2} \\
& =c \sum_{\tau / 2<j \leq \tau}\left(\sum_{n \in \mathcal{L}_{j}} d_{n}^{2}\right)^{1 / 2} .
\end{aligned}
$$

The upper bound immediately follows from the Cauchy-Schwarz inequality.

Corollary 3.3. If $\left(d_{n}\right)$ is a multiplicative system, we have

$$
\mathbb{E} \sup _{\underline{z} \in \mathcal{Z}}\left|Q^{\prime}(\underline{z})\right| \geq c \sum_{\tau / 2<j \leq \tau} d_{p_{j}}\left(\sum_{\substack{\tilde{n} \leq N / p_{j} \\ P^{+}(\widetilde{n}) \leq p_{\tau / 2}}} d_{\widetilde{n}}^{2}\right)^{1 / 2} .
$$

Now we can finish the proof of Theorem 1.1. 
Proof of the lower bound in Theorem 1.1. If $d_{n} \equiv n^{-\sigma}$, the above corollary yields

$$
\begin{aligned}
\mathbb{E} \sup _{\underline{z} \in \mathbb{T}^{\tau}}\left|\sum_{j=1}^{\tau} \sum_{n \in E_{j}} \varepsilon_{n} n^{-\sigma} e^{2 i \pi\langle\underline{a}(n), \underline{z}\rangle}\right| \geq \mathbb{E} \sup _{\underline{z} \in \mathcal{Z}}\left|Q^{\prime}(\underline{z})\right| \\
\geq \frac{C}{N^{\sigma}} \sum_{\tau / 2<j \leq \tau} \#\left\{m \leq N / p_{j}: P^{+}(m) \leq p_{\tau / 2}\right\}^{1 / 2} \\
=\frac{C}{N^{\sigma}} \sum_{\tau / 2<j \leq \tau} \Psi\left(N / p_{j}, p_{\tau / 2}\right)^{1 / 2} .
\end{aligned}
$$

Since

$$
\begin{aligned}
\Psi\left(\frac{N}{p_{j}}, p_{\tau / 2}\right) & \geq \Psi\left(\frac{N}{p_{\tau}}, p_{\tau / 2}\right)=\frac{N}{p_{\tau}} \Psi^{*}\left(\frac{N}{p_{\tau}}, p_{\tau / 2}\right) \\
& \geq \frac{c N}{\tau \log \tau} \Psi^{*}\left(\frac{N}{p_{\tau}}, p_{\tau / 2}\right),
\end{aligned}
$$

we obtain

$$
\begin{aligned}
\mathbb{E} \sup _{\underline{z} \in \mathbb{T}^{\tau}}\left|\sum_{j=1}^{\tau} \sum_{n \in E_{j}} \varepsilon_{n} n^{-\sigma} e^{2 i \pi\langle\underline{a}(n), \underline{z}\rangle}\right| & \geq \frac{c}{N^{\sigma}} \frac{\tau}{2}\left[\frac{c N}{\tau \log \tau} \Psi^{*}\left(\frac{N}{p_{\tau}}, p_{\tau / 2}\right)\right]^{1 / 2} \\
& =c N^{1 / 2-\sigma}\left(\frac{\tau}{\log \tau}\right)^{1 / 2} \Psi^{*}\left(\frac{N}{p_{\tau}}, p_{\tau / 2}\right)^{1 / 2}
\end{aligned}
$$

as asserted.

4. Proof of Theorem 1.2. We need to prove the upper bound, since the lower bound was obtained in Theorem 1.1. Moreover, we are only going to show how the calculations concerning the upper bound of Theorem 1.1 should be corrected in order to get an extra Dickman-type factor.

STEP 1. Some remarks on a semi-asymptotic formula for the Dickman function. We discuss the so called semi-asymptotic formula (see [BT])

$$
\Psi(a x, y)=a^{\alpha(x, y)} \Psi(x, y)(1+O(1 / \bar{u}))
$$

where $\bar{u}=\min \{\log x, y\} / \log y$ and

$$
\begin{aligned}
\alpha(x, y) & =\frac{\log (1+y / \log x)}{\log y}=1-\frac{\log \log x}{\log y}+\frac{\log (1+\log x / y)}{\log y} \\
& =1-\frac{\log \log x}{\log y}+O\left(\frac{\log x}{y \log y}\right) .
\end{aligned}
$$

Since in our zone $y>\log x$, we have

$$
O\left(\frac{\log x}{y}\right)=O(1)=o(\log \log x)
$$


Therefore $\alpha \leq 1$ for $x$ large enough. We also see that $\alpha \rightarrow 1$ as $x \rightarrow \infty$, hence $\alpha \geq 2 / 3$ for all $x$ large enough. In what follows, we assume that $2 / 3 \leq \alpha \leq 1$.

STEP 2: Main estimate and adjustment of the previous proof. We still use the notation $\Psi^{*}(x, y)=x^{-1} \Psi(x, y)$ but skip $y$ everywhere since $y=p_{\nu}$. In other words, we write $\Psi(x):=\Psi\left(x, p_{\nu}\right)$ and $\Psi^{*}(x):=\Psi^{*}\left(x, p_{\nu}\right)$.

Let $b^{*}=1$ for $b=1$ and $b^{*}=2 b / 3$ for $b=2,3, \ldots$ We will prove that for all $b_{1}, b_{2} \geq 1, j_{1}, j_{2} \leq \nu$,

$$
\Psi\left(\frac{N}{p_{j_{1}}^{b_{1}} p_{j_{2}}^{b_{2}}}\right) \leq C \frac{N}{p_{j_{1}}^{b_{1}^{*}} p_{2}^{b_{2}^{*}}} \Psi^{*}\left(\frac{N}{p_{\nu}^{2}}\right) .
$$

Once (4.2) is proved, the calculation in (2.6) is updated as follows. Let $D_{j}=\left|z_{j}-z_{j}^{\prime}\right|$. Then

$$
\begin{aligned}
d\left(z, z^{\prime}\right)^{2} & \leq C \sum_{j_{1}, j_{2} \leq \nu} D_{j_{1}} D_{j_{2}} \sum_{b_{1}, b_{2}=1}^{\infty} b_{1} b_{2} p_{j_{1}}^{-2 b_{1} \sigma} p_{j_{2}}^{-2 b_{2} \sigma} \Psi\left(\frac{N}{p_{j_{1}}^{b_{1}} p_{j_{2}}^{b_{2}}}\right)\left(\frac{N}{p_{j_{1}}^{b_{1}} p_{j_{2}}^{b_{2}}}\right)^{-2 \sigma} \\
& =C N^{-2 \sigma} \sum_{j_{1}, j_{2} \leq \nu} D_{j_{1}} D_{j_{2}} \sum_{b_{1}, b_{2}=1}^{\infty} b_{1} b_{2} \Psi\left(\frac{N}{p_{j_{1}}^{b_{1}} p_{j_{2}}^{b_{2}}}\right) \\
& \leq C N^{1-2 \sigma} \Psi^{*}\left(\frac{N}{p_{\nu}^{2}}\right) \sum_{j_{1}, j_{2} \leq \nu} D_{j_{1}} D_{j_{2}} \sum_{b_{1}, b_{2}=1}^{\infty} b_{1} b_{2} p_{j_{1}}^{-b_{1}^{*}} p_{j_{2}}^{-b_{2}^{*}} \\
& =C N^{1-2 \sigma} \Psi^{*}\left(\frac{N}{p_{\nu}^{2}}\right)\left\{\sum_{j \leq \nu} D_{j} \sum_{b=1}^{\infty} b p_{j}^{-b^{*}}\right\}^{2} .
\end{aligned}
$$

Now everything continues as in the proof of Theorem 1.1 but with an extra factor $\Psi^{*}\left(N / p_{\nu}^{2}\right)$. The minor change (corresponding to $\left.(2.9)\right)$ is that

$$
\sum_{b=2}^{\infty} b p_{j}^{-b^{*}}=\sum_{b=2}^{\infty} b\left(\frac{2}{p_{j}}\right)^{b^{*}} 2^{-b^{*}} \leq\left(\frac{2}{p_{j}}\right)^{4 / 3} \sum_{b=2}^{\infty} b 2^{-b^{*}}=\frac{C}{p_{j}^{4 / 3}},
$$

hence still

$$
d_{1} \leq \sum_{j=1}^{\nu} \frac{C}{p_{j}^{4 / 3}} \max _{j} D_{j} \leq C \varepsilon .
$$

STEP 3: The proof of inequality (4.2). We consider three cases:

1. $b_{1}, b_{2} \geq 2$. By applying (4.1) with $x=N / p_{j_{1}}^{b_{1}} p_{j_{2}}^{b_{2}}$ and $a=p_{j_{1}}^{b_{1}} p_{j_{2}}^{b_{2}}$, we get

$$
\Psi(N)=\left(p_{j_{1}}^{b_{1}} p_{j_{2}}^{b_{2}}\right)^{\alpha} \Psi\left(\frac{N}{p_{j_{1}}^{b_{1}} p_{j_{2}}^{b_{2}}}\right)(1+O(1 / \bar{u}))
$$


Once $\bar{u}$ is large enough and $\alpha \geq 2 / 3$ we have

$$
\Psi\left(\frac{N}{p_{j_{1}}^{b_{1}} p_{j_{2}}^{b_{2}}}\right) \leq C \Psi(N)\left(p_{j_{1}}^{b_{1}} p_{j_{2}}^{b_{2}}\right)^{-2 / 3}
$$

Similarly, we pass from $\Psi(N)$ to $\Psi\left(N / p_{\nu}^{2}\right)$. By using $\alpha \leq 1$, we have

$$
\Psi(N)=\left[p_{\nu}^{2}\right]^{\alpha} \Psi\left(\frac{N}{p_{\nu}^{2}}\right)(1+O(1 / \bar{u})) \leq C p_{\nu}^{2} \Psi\left(\frac{N}{p_{\nu}^{2}}\right)=C N \Psi^{*}\left(\frac{N}{p_{\nu}^{2}}\right)
$$

By combining the two estimates we get

$$
\Psi\left(\frac{N}{p_{j_{1}}^{b_{1}} p_{j_{2}}^{b_{2}}}\right) \leq C\left(p_{j_{1}}^{b_{1}} p_{j_{2}}^{b_{2}}\right)^{-2 / 3} N \Psi^{*}\left(\frac{N}{p_{\nu}^{2}}\right)
$$

as required.

2. $b_{1}=b_{2}=1$. By applying (4.1) with $x=N / p_{j_{1}} p_{j_{2}}$ and $a=p_{\nu}^{2} / p_{j_{1}} p_{j_{2}}$, we get, using $\alpha \leq 1$,

$$
\begin{aligned}
\Psi\left(\frac{N}{p_{j_{1}} p_{j_{2}}}\right) & \leq C\left(\frac{p_{\nu}^{2}}{p_{j_{1}} p_{j_{2}}}\right)^{\alpha} \Psi\left(\frac{N}{p_{\nu}^{2}}\right) \leq C \frac{p_{\nu}^{2}}{p_{j_{1}} p_{j_{2}}} \Psi\left(\frac{N}{p_{\nu}^{2}}\right) \\
& =C \frac{N}{p_{j_{1}} p_{j_{2}}} \Psi^{*}\left(\frac{N}{p_{\nu}^{2}}\right)
\end{aligned}
$$

as required.

3. $b_{1}=1, b_{2} \geq 2$. By applying (4.1) with $x=N / p_{j_{1}} p_{j_{2}}^{b_{2}}$ and $a=p_{j_{2}}^{b_{2}}$, we get, using $\alpha \geq 2 / 3$,

$$
\Psi\left(\frac{N}{p_{j_{1}}}\right)=\left(p_{j_{2}}^{b_{2}}\right)^{\alpha} \Psi\left(\frac{N}{p_{j_{1}} p_{j_{2}}^{b_{2}}}\right)(1+O(1 / \bar{u}))
$$

hence

$$
\Psi\left(\frac{N}{p_{j_{1}} p_{j_{2}}^{b_{2}}}\right) \leq C p_{j_{2}}^{-2 b_{2} / 3} \Psi\left(\frac{N}{p_{j_{1}}}\right)=C p_{j_{2}}^{-b_{2}^{*}} \Psi\left(\frac{N}{p_{j_{1}}}\right)
$$

Yet, letting $p_{j_{2}}=1$ in (4.3), we have

$$
\Psi\left(\frac{N}{p_{j_{1}}}\right) \leq C \frac{N}{p_{j_{1}}} \Psi^{*}\left(\frac{N}{p_{\nu}^{2}}\right),
$$

and we are done. Therefore, the proof of (4.2) is complete.

We finish the section by giving a proof of $\left(1.4^{\prime}\right)$. Only the upper bound needs a proof. Fix some large integer $M$. Let $\left\{g_{n}, n \geq 1\right\}$ be a sequence of independent $\mathcal{N}(0,1)$ distributed random variables. By the contraction principle, there is an absolute constant $C$ such that

$$
\mathbb{E} \sup _{N \leq M} \sup _{t \in \mathbb{R}} \frac{\left|\sum_{n=2}^{N} \varepsilon_{n} n^{-\sigma-i t}\right|}{N^{1-\sigma}(\log N)^{-1}} \leq C \mathbb{E} \sup _{N \leq M} \sup _{t \in \mathbb{R}} \frac{\left|\sum_{n=2}^{N} g_{n} n^{-\sigma-i t}\right|}{N^{1-\sigma}(\log N)^{-1}} .
$$


We now need the following inequality (see [W1, p. 451]), which is a simple consequence of the Borell-Sudakov-Tsirelson inequality: if $G_{1}, \ldots, G_{N}$ are Gaussian random vectors with values in a separable Banach space $(B,\|\cdot\|)$, then

$$
\mathbb{E} \sup _{1 \leq k \leq N}\left\|G_{k}\right\| \leq C\left\{\sup _{1 \leq k \leq N} \mathbb{E}\left\|G_{k}\right\|+\mathbb{E} \sup _{1 \leq k \leq N} \sigma_{k}\left|\zeta_{k}\right|\right\}
$$

where $\sigma_{k}=\sup _{f \in B^{*},\|f\| \leq 1}\left(\mathbb{E}\left\langle f, G_{k}\right\rangle^{2}\right)^{1 / 2}, k=1, \ldots, N,\left\{\zeta_{k}, 1 \leq k \leq N\right\}$ is a sequence of independent $\mathcal{N}(0,1)$ distributed random variables, and $C$ is a universal constant.

Applying this inequality gives

$$
\begin{aligned}
\mathbb{E} \sup _{N \leq M} \sup _{t \in \mathbb{R}} \frac{\left|\sum_{n=2}^{N} g_{n} n^{-\sigma-i t}\right|}{N^{1-\sigma}(\log N)^{-1}} \\
\quad \leq C \sup _{N \leq M} \mathbb{E} \sup _{t \in \mathbb{R}} \frac{\left|\sum_{n=2}^{N} g_{n} n^{-\sigma-i t}\right|}{N^{1-\sigma}(\log N)^{-1}}+C \mathbb{E} \sup _{N \leq M}\left|\zeta_{N} \sigma_{N}\right| \\
\leq C_{\sigma}+C \mathbb{E} \sup _{N \leq M}\left|\zeta_{N} \sigma_{N}\right|,
\end{aligned}
$$

where

$$
\begin{aligned}
\sigma_{N} & \leq C \frac{\sup _{t \in \mathbb{R}}\left\|\sum_{n=2}^{N} g_{n} n^{-\sigma-i t}\right\|_{2}}{N^{1-\sigma}(\log N)^{-1}} \leq C \frac{\left(\sum_{n=2}^{N} n^{-2 \sigma}\right)^{1 / 2}}{N^{1-\sigma}(\log N)^{-1}} \\
& \leq C_{\sigma} \frac{N^{1 / 2-\sigma}}{N^{1-\sigma}(\log N)^{-1}}=C_{\sigma} \frac{\log N}{N^{1 / 2}} .
\end{aligned}
$$

It is obvious that $\mathbb{E} \sup _{N<M}\left(\left|\zeta_{N}\right| \log N\right) / N^{1 / 2}$ is bounded uniformly in $M$ by some absolute constant. So there exists a constant $C_{\sigma}$, depending on $\sigma$ only, such that for any $M$,

$$
\mathbb{E} \sup _{N \leq M} \sup _{t \in \mathbb{R}} \frac{\left|\sum_{n=2}^{N} g_{n} n^{-\sigma-i t}\right|}{N^{1-\sigma}(\log N)^{-1}} \leq C_{\sigma} .
$$

The claimed result follows immediately.

Note to conclude that the same argument applies to our upper bounds results with minor modifications (by introducing suitable blocks).

5. Other results. In this section we test our technique on some other sets of coefficients.

Let $\left\{d_{n}, n \geq 1\right\}$ be a sequence of multiplicative weights: $d_{n m}=d_{n} d_{m}$ whenever $n, m$ are coprime. Set

$$
B_{m}=\sum_{2 \leq n \leq m} d_{n}^{2}
$$


By choosing $\tau=\mu:=\pi(N)$ in the lower bound of Proposition 3.2, we get

$$
\begin{aligned}
\mathbb{E} \sup _{\underline{z} \in \mathbb{T}^{\mu}}\left|\sum_{n=2}^{N} d_{n} \varepsilon_{n} n^{-\sigma} e^{2 i \pi\langle\underline{a}(n), \underline{z}\rangle}\right| & \\
& \geq \mathbb{E} \sup _{\underline{z} \in \mathcal{Z}}\left|Q^{\prime}(\underline{z})\right| \geq C N^{-\sigma} \sum_{\mu / 2<j \leq \mu} d_{p_{j}}\left(\sum_{\substack{\tilde{n} \leq N / p_{j} \\
P^{+}(\widetilde{n}) \leq p_{\mu / 2}}} d_{\widetilde{n}}^{2}\right)^{1 / 2} .
\end{aligned}
$$

Note that for large $N$ in the case $\tau=\mu$ the sets $\mathcal{L}_{j}$ reduce to $\left\{n=p_{j} \widetilde{n}\right.$ : $\left.\widetilde{n} \leq N / p_{j}\right\}$. Indeed, if $\widetilde{n} \leq N / p_{j}$ and if there is an $s \geq \mu / 2$ such that $p_{s} \mid \widetilde{n}$, then this implies that

$$
N \geq p_{j} p_{s} \geq p_{\mu / 2}^{2} \sim(\mu \log \mu)^{2} / 4 \sim N^{2} / 4,
$$

which is impossible for large $N$. Thus necessarily $P^{+}(\widetilde{n}) \leq p_{\mu / 2}$. Hence

We have obtained

$$
\begin{aligned}
\mathbb{E} \sup _{\underline{z} \in \mathbb{T}^{\mu}} \mid \sum_{n=2}^{N} d_{n} \varepsilon_{n} n^{-\sigma} e^{2 i \pi\langle\underline{a}(n), \underline{z}\rangle \mid} & \geq C N^{-\sigma} \sum_{\mu / 2<j \leq \mu} d_{p_{j}}\left(\sum_{\widetilde{n} \leq N / p_{j}} d_{\tilde{n}}^{2}\right)^{1 / 2} \\
& =C N^{-\sigma} \sum_{\mu / 2<j \leq \mu} d_{p_{j}} B_{N / p_{j}}^{1 / 2} .
\end{aligned}
$$

Proposition 3.4. There exists a universal constant $C$ and $N_{0}$ such that for any $0 \leq \sigma<1 / 2$, any integer $N \geq N_{0}$ and any multiplicative sequence of weights $\left(d_{n}\right)$,

$$
\mathbb{E} \sup _{t \in \mathbb{R}}\left|\sum_{n=2}^{N} \varepsilon_{n} d_{n} n^{-\sigma-i t}\right| \geq C N^{-\sigma} \sum_{\mu / 2<j \leq \mu} d_{p_{j}} B_{N / p_{j}}^{1 / 2},
$$

where $B_{m}$ is defined in (5.1).

Apply this to the case $d_{n}=d(n)$, where $d(n)=\sharp\{d: d \mid n\}$ is the divisor function. Although these weights are very irregular, their sums behave regularly, in particular,

$$
\sum_{n=1}^{N}(d(n))^{2} \sim \frac{N}{\pi^{2}}(\log N)^{3}
$$

as $N$ tends to infinity. The last estimate immediately provides $B_{m} \sim$ $\left(m / \pi^{2}\right) \log ^{3} m$, hence (noticing that $d_{p_{j}}=2$ and $\mu \sim N / \log N$ )

$$
\begin{aligned}
\sum_{\mu / 2<j \leq \mu} d_{p_{j}} B_{N / p_{j}}^{1 / 2} & \sim \sum_{\mu / 2<j \leq \mu}\left(2 N / p_{j} \pi^{2}\right)^{1 / 2}\left(\log \frac{N}{p_{j}}\right)^{3 / 2} \\
& =\frac{2 N^{1 / 2}}{\pi} \sum_{\mu / 2<j \leq \mu} \frac{1}{p_{j}^{1 / 2}}\left(\log \frac{N}{p_{j}}\right)^{3 / 2}
\end{aligned}
$$




$$
\begin{aligned}
& \sim \frac{2 N^{1 / 2}}{\pi} \sum_{\mu / 2<j \leq \mu} \frac{\left(\log \frac{N}{j \log j}\right)^{3 / 2}}{(j \log j)^{1 / 2}} \approx N^{1 / 2} \sum_{\mu / 2<j \leq \mu} \frac{1}{(j \log j)^{1 / 2}} \\
& \approx N^{1 / 2} \frac{\mu^{1 / 2}}{(\log \mu)^{1 / 2}} \sim \frac{N}{\log N} .
\end{aligned}
$$

Now, let $\left\{P_{k}: k \in K\right\}$ be a finite set of mutually coprime numbers. Consider the set of integers

$$
E=\left\{n: n=\prod_{k \in K} P_{k}^{\alpha_{k}}, \alpha_{k} \in\{0,1\}\right\}
$$

and the associated Dirichlet polynomial

$$
D_{E}(t)=\sum_{n \in E} \varepsilon_{n} n^{-\sigma-i t}=\sum_{n=2}^{N} \varepsilon_{n} \chi_{E}(n) n^{-\sigma-i t},
$$

where $N=\prod_{k \in K} P_{k}$. We prove the following.

Proposition 3.5. There exists a universal constant $C$ such that, for any $\sigma \geq 0$ and any $\left\{P_{k}: k \in K\right\}$,

$$
\mathbb{E} \sup _{t \in \mathbb{R}}\left|D_{E}(t)\right| \geq C \prod_{k \in K}\left(1+P_{k}^{-2 \sigma}\right)^{1 / 2} \sup _{G \subseteq K} \frac{\sum_{j \in G} P_{j}^{-\sigma}}{\prod_{k \in G}\left(1+P_{k}^{-2 \sigma}\right)^{1 / 2}}
$$

Proof. By (1.1) we have

$$
\sup _{t \in \mathbb{R}}\left|D_{E}(t)\right|=\sup _{\underline{z} \in \mathbb{T}^{\mu}}|Q(\underline{z})|
$$

where $\mu=|K|$ and

$$
Q(\underline{z})=\sum_{n=2}^{N} \chi_{E}(n) \varepsilon_{n} n^{-\sigma} e^{2 i \pi\langle\underline{a}(n), \underline{z}\rangle} .
$$

Let $A \subset K$ and $B=K \backslash A$. We assume that both $A$ and $B$ are nonempty sets. For $j \in B$, define

$$
\mathcal{B}_{j}=\left\{n \in E: \alpha_{k}=0 \text { if } k \in B, k \neq j, \text { and } \alpha_{j}=1\right\}
$$

and $\mathcal{Z} \subset \mathbb{T}^{\mu}$ by

$$
\mathcal{Z}=\left\{\underline{z}=\left\{z_{k}, 1 \leq k \leq 2 r\right\}: z_{k}=0 \text { if } k \in A \text {, and } z_{k} \in\{0,1 / 2\} \text { if } k \in B\right\} .
$$

For $j \in B, n \in \mathcal{B}_{j}$ and $z \in \mathcal{Z}$, we have $2\langle\underline{a}(n), \underline{z}\rangle=2 \sum_{k \in K} \alpha_{k} z_{k}=2 z_{j}=$ \pm 1 , so that similarly to our previous lower bound,

$$
\sup _{\underline{z} \in \mathcal{Z}}|Q(\underline{z})| \geq \sum_{j \in B}\left|\sum_{n \in \mathcal{B}_{j}} \varepsilon_{n} n^{-\sigma}\right|
$$


almost surely. Hence

$$
\begin{aligned}
\mathbb{E} \sup _{\underline{z} \in \mathcal{Z}}|Q(\underline{z})| & \geq C \sum_{j \in B}\left(\mathbb{E}\left|\sum_{n \in \mathcal{B}_{j}} \varepsilon_{n} n^{-2 \sigma}\right|^{2}\right)^{1 / 2} \\
& =C \sum_{j \in B} P_{j}^{-\sigma}\left(\sum_{\left(\alpha_{k}\right)_{k \in A} \in\{0,1\}^{A}} \prod_{k \in A} P_{k}^{-2 \sigma \alpha_{k}}\right)^{1 / 2} \\
& =C \prod_{k \in A}\left(1+P_{k}^{-2 \sigma}\right)^{1 / 2}\left\{\sum_{j \in B} P_{j}^{-\sigma}\right\}
\end{aligned}
$$

Therefore

$$
\begin{aligned}
\mathbb{E} \sup _{t \in \mathbb{R}}\left|D_{E}(t)\right| \geq C \sup _{A \subseteq K, A \neq K} \prod_{k \in A}\left(1+P_{k}^{-2 \sigma}\right)^{1 / 2}\left\{\sum_{j \in A^{\mathrm{c}}} P_{j}^{-\sigma}\right\} \\
=C \prod_{k \in K}\left(1+P_{k}^{-2 \sigma}\right)^{1 / 2} \sup _{A \subseteq K, A \neq K} \frac{\sum_{j \in A^{\mathrm{c}}} P_{j}^{-\sigma}}{\prod_{k \in A^{\mathrm{c}}}\left(1+P_{k}^{-2 \sigma}\right)^{1 / 2}} .
\end{aligned}
$$

Acknowledgements. The work of the first mentioned author was supported by grants RFBR 05-01-00911 and INTAS 03-51-5018. He is also grateful for the hospitality of the L. Pasteur University (Strasburg) where this research has been done.

\section{References}

[BKQ] F. Bayart, S. V. Konyagin and H. Queffélec, Convergence almost everywhere and divergence almost everywhere of Taylor and Dirichlet series, Real Anal. Exchange 29 (2003/2004), 557-586.

[BH] H. F. Bohnenblust and E. Hille, On the absolute convergence of Dirichlet series, Ann. of Math. 32 (1931), 600-622.

[B] H. Bohr, Collected Mathematical Works, Copenhagen, 1952.

[BT] R. de la Bretèche and G. Tenenbaum, Entiers friables: inégalité de TuránKubilius et applications, Invent. Math. 159 (2005), 531-588.

[C] L. E. Clarke, Dirichlet series with independent and identically disturbed coeffcients, Proc. Cambridge Philos. Soc. 66 (1969), 393-397.

[DC] A. Dvoretzky et H. Chojnacki, Sur les changements de signe d'une série à termes complexes, C. R. Acad. Sci. Paris 222 (1947), 515-518.

[DE1] A. Dvoretzky and P. Erdős, On power series diverging everywhere on the circle of convergence, Michigan Math. J. 3 (1955), 31-35.

[DE2] -, 一, Divergence of random power series, Michigan Math. J. 6 (1959), 343-347.

$[\mathrm{H}] \quad$ H. Helson, Foundations of the theory of Dirichlet series, Acta Math. 118 (1967), $61-77$.

[Ha1] G. Halász, private communication to H. Queffélec, see [Q1].

[Ha2] - On random multiplicative functions, in: Hubert Delange Colloquium (Orsay 1982), Publ. Math. Orsay 83-4, Univ. Paris XI, Orsay, 1983, 74-96. 
[HR] G. H. Hardy and M. Riesz, The General Theory of Dirichlet's Series, Cambridge Tracts in Math. and Math. Phys. 18, Cambridge Univ. Press, 1915.

[HW] G. H. Hardy and E. M. Wright, An Introduction to the Theory of Numbers, 5th ed., Oxford Univ. Press, Clarendon Press, 1979.

[Har] P. Hartman, On Dirichlet series involving random coefficients, Amer. J. Math. 61 (1939), 955-964.

[HS] H. Hedenmalm and E. Saksman, Carleson's convergence theorem for Dirichlet series, Pacific J. Math. 208 (2003), 85-109.

[K] J. P. Kahane, Some Random Series of Functions, D. C. Heath and Co. Raytheon Education Co., Lexington, MA, 1968.

[KS] B. S. Kashin and A. A. Saakyan, Orthogonal Series, Transl. Math. Monogr. 75, Amer. Math. Soc., 1989.

[KQ] S. V. Konyagin and H. Queffélec, The translation $\frac{1}{2}$ in the theory of Dirichlet series, Real Anal. Exchange 27 (2001/2002), 155-176.

[L] M. A. Lifshits, Gaussian Random Functions, Kluwer, Dordrecht, 1995.

[PSW] G. Peskir, D. Schneider and M. Weber, Randomly weighted series of contractions in Hilbert spaces, Math. Scand. 79 (1996), 263-282.

[Q1] H. Queffélec, Propriétés presque sûres et quasi-sûres des séries de Dirichlet et des produits d'Euler, Canad. J. Math. 32 (1980), 531-558.

[Q2] - Sur une estimation probabiliste liée à l'inégalité de Bohr, in: Harmonic Analysis: Study Group on Translation-Invariant Banach Spaces, Publ. Math. Orsay 84-1, exp. no. 6, Univ. Paris XI, Orsay, 1984, 21 pp.

[Q3] - $\quad$ H. Bohr's vision of ordinary Dirichlet series; old and new results, J. Anal. 3 (1995), 43-60.

[STY] D. Sun, F. Tian and J. R. Yu, Sur les séries aléatoires de Dirichlet, C. R. Acad. Sci. Paris Sér. I 326 (1998), 427-431.

[T] G. Tenenbaum, Introduction à la théorie analytique et probabiliste des nombres, Revue de l'Institut Élie Cartan 13, Département de Mathématiques de l'Université de Nancy I, 1990.

[W1] M. Weber, Estimating random polynomials by means of metric entropy methods, Math. Inequal. Appl. 3 (2000), 443-457.

[W2] - On a stronger form of Salem-Zygmund's inequality for random trigonometric sums with examples, Period. Math. Hungar. 52 (2006), 73-104.

[Y1] J. R. Yu, Some properties of random Dirichlet series, Acta Math. Sinica 21 (1978), 97-118.

[Y2] - Sur quelques séries gaussiennes de Dirichlet, C. R. Acad. Sci. Paris Sér. I 300 (1985), 521-522.

[Y3] —, Dirichlet spaces and random Dirichlet series, J. Anal. 3 (1995), 61-71.

Department of Mathematics and Mechanics

St. Petersburg State University

Bibliotechnaya pl. 2

198504, Stary Peterhof, Russia

E-mail: lifts@mail.rcom.ru
Mathématique (IRMA) Université Louis-Pasteur et C.N.R.S.

7 rue René Descartes 67084 Strasbourg Cedex, France E-mail: weber@math.u-strasbg.fr 